\title{
Anforderungen an die klinische Therapieforschung in der Phase I, II und III
}

\author{
L. Edler \\ ZE Biostatistik, Deutsches Krebsforschungszentrum, Heidelberg
}

\section{Einleitung}

Für die Verbesserung der Behandlung von Krebspatienten sind optimale Therapiekonzepte in einem schrittweisen experimentell orientierten Vorgehen zu entwickeln, um sie nach ausreichender Prüfung in der Patientenbehandlung und -versorgung anzuwenden. Entwicklung und Prüfung erfolgen in klinischen Studien. Aus patientenbezogenen klinischen Daten werden - mittels statistischer Verfahren - Schlussfolgerungen zur Wirksamkeit, Dosierung und Sicherheit von Behandlungen gezogen [vergleiche z.B. 1-3].

In der Onkologie, aber auch in anderen medizinischen Forschungsbereichen wie z.B. der Kardiologie, der Rheumatologie oder der AIDS-Forschung hat sich eine geordnete Abfolge von Studien in den Phasen I-III bewährt: Ausgehend von einer Dosisfindung in der Phase I nähert man sich über einen grundsätzlichen Wirkungsnachweis in der Phase II und dem randomisierten Wirksamkeitsvergleich in der Phase III einer optimalen Behandlung. Dieser sequentielle Zugang findet sich in modifizierter Form in einem sequentiellen Vorgehen in den einzelnen Studienphasen wieder. Ethische Anforderungen an klinische Studien, die in den Forderungen der Deklaration von Helsinki niedergelegt sind, haben dieses sequentielle Vorgehen wesentlich begründet.

In den folgenden drei Kapiteln werden die wesentlichen methodischen Anforderungen formuliert. Zunächst werden grundsätzliche Voraussetzungen für klinische Studien dargelegt. Diese werden dann erweitert und verfeinert auf die drei Phasen I, II und III. Schließlich werden Qualitätssicherung und neuere Studienkonzepte diskutiert.

\section{Anforderungen an klinische Studien}

Eine klinischen Studie gliedert sich nach Ablauf und methodischen Gesichtspunkten grundsätzlich in vier Abschnitte:

1. Planung: Studiendesign, Formulierung der Fragestellung, Ableitung der Prüfhypothesen, Festlegung einer ausreichenden Fallzahl, Methodische Maßnahmen zur Qualitätssicherung.

2. Durchführung: Behandlung, Verlaufskontrolle, Nachsorge, Monitoring, Qualitätskontrolle der Patientendaten, Zwischenauswertungen, Studiensitzungen.

3. Auswertung: statistische Datenanalyse mit einem konfirmatorischen (Signifikanztest, p-Wert, Konfidenzintervall) und mit einem explorativen Teil (Tabellen, Grafiken, Regressionsmodelle) mit Aussagen zu Wirksamkeit, Sicherheit und Prognose.

4. Bericht und Publikation: Interpretation der Ergebnisse.

\section{Anforderung an die Planung}

Die Planung einer klinischen Studie sollte durch zwei Qualitätsprinzipien geleitet sein: das Erreichen von Genauigkeit und die Vermeidung von Fehlern. Die Genauigkeitsforderung hat Konsequenzen für die Anzahl der Fälle. Die zweite Forderung zielt darauf, mögliche Verzerrungen zu vermeiden, und hat Konsequenzen für die Durchführung einer Studie. Eine gut geplante klinische Studie hält sich an ein prospektiv festgelegtes Studienprotokoll, welches alle Einzelheiten der Durchführung des Forschungsprojekts und die Behandlung aller Patienten festlegt. Nicht zuletzt bedingt durch eine weitreichende behördliche Regulation, sind Studienprotokolle inzwischen in der klinischen Forschung sehr umfangreich geworden und gaben Anlass zu behördlicher Regulation (Richtlinien: www.eudra.org; www.fda.gov/cder/guidance/index.htm;

\begin{tabular}{ll}
\hline KARGER & ( ) 2002 S. Karger GmbH, Freiburg \\
Fax +497614520714 & Accessible online at: \\
$\begin{array}{l}\text { E-mail Information@Karger.de } \\
\text { www.karger.com }\end{array}$ & www.karger.com/journals/onk
\end{tabular}

Dr. Lutz Edler

ZE Biostatistik R0700, Deutsches Krebsforschungszentrum

Im Neuenheimer Feld 280, D-69120 Heidelberg (Deutschland)

Tel./Fax +496221 $422392 / 2397$

E-mailedler@dkfz.de 
www.ifpma.org/ich5.html). Wenige aber essentielle Punkte bestimmen ein Studienprotokoll und die Güte einer Studie:

1. die Darstellung der Ziele, die zu prüfenden Hypothesen und die relevanten Zielgrößen;

2. die Ein-/Ausschlusskriterien zur Definition der Patientenpopulation;

3. die Beschreibung aller (Be-)Handlungen;

4. die Beschreibung aller Messungen und Beobachtungen einschließlich Toxizität und unerwünschter Ereignisse («adverse events»);

5. die Angaben zum biometrischen Design, Fallzahlplanung und Auswerteplan;

6. die Beschreibung von Maßnahmen der Qualitätssicherung.

\section{Anforderung an die Festlegung der relevanten Zielgröße}

Mit der Zielgröße wird der relevante Behandlungseffekt gemessen. Ihre Wahl ist maßgeblich für den Studienerfolg, weswegen sie auch prospektiv eindeutig definiert sein muss. Treten bei der Beurteilung der Zielgröße Meinungsunterschiede oder Interpretationsschwierigkeiten auf (z.B. beim Ansprechen), so ist eine möglichst objektive und unvoreingenommene (Referenz-)Beurteilung einzuholen, die bevorzugt blind gegenüber der Behandlung erfolgen sollte. Aus rein pragmatischen Gründen hat es sich bewährt, Zielgrößen in primäre, sekundäre und erklärende zu unterteilen, um eine Fallzahlerhöhung zu vermeiden, die sonst wegen Mehrfachvergleichen und dem damit verbundenen Anstieg des Fehlers 1. Art unumgänglich wird.

Die Überlebenszeit nach Eintritt einer Erkrankung ist eine objektive und in der Regel einfach zu bestimmende Zielgröße, allerdings meist nur in der Phase-III-Studie als Hauptzielgröße. Man beachte, dass die Macht einer Überlebenszeit-Studie von der Anzahl der Sterbefälle bestimmt ist. Für die Planung der Studie ist somit nicht die Anzahl der ordnungsgemäß aufgenommenen Patienten, sondern die Anzahl der Ereignisse ausschlaggebend, die im Laufe der Studie beobachtet werden. Eine Nachforschung der Überlebenszeit für möglichst alle auswertbaren Patienten ist deswegen unerlässlich, und eine zu frühe Auswertung der Daten ist zu unterlassen. Für die Auswertung der Überlebenskurven sind die anerkannten Methoden nach Kaplan-Meier und das Regressionsmodell von Cox anzuwenden, welches die zeitabhängige Sterberate eines Patienten in Abhängigkeit von Behandlung und Prognosefaktoren berechnet.

Ein grundsätzliches Problem der Beurteilung der Lebensqualität als Zielgröße ist deren Subjektivität. Als primäre Zielgröße ist für ihre Gültigkeit eine Verblindung unumgänglich, um einen Einfluss der Kenntnis der Behandlung auf das Ergebnis auszuschließen. Allerdings stößt in onkologischen Studien das Prinzip der Verblindung rasch an ethische und auch praktische Grenzen, da eine Therapie vom Toxizitätsspektrum her oft leicht erraten werden kann und da der Arzt die Patienten eines Armes wegen der Möglichkeit schwerwiegender Toxizitäten unter Umständen besonders im Auge behalten muss und insbesondere über mögliche Toxizität aufzuklären hat. Diese Schwierigkeiten einer Verblindung werden oft unterschätzt und erschweren die Verwendung der Lebensqualität als Hauptzielgröße.

\section{Anforderungen an die Logistik und die Studienzentrale}

Um die Fallzahl zu erhöhen, aber auch aus Gründen der Verallgemeinerungsfähigkeit, dem zeitnahen Erreichen des Ergebnisses, der schnellen Verbreitung neuer Therapien und ihrer Akzeptanz werden heute Patienten aus mehreren Zentren in eine Studie aufgenommen. Eine multizentrische Organisationsstruktur muss gewährleisten, dass in jedem Zentrum das Studienprotokoll eingehalten wird und somit die Patienten der Studie insgesamt vergleichbar sind. Eine Studienleitung übernimmt die Gesamtverantwortung für die Studie und die Koordination aller Zentren und weiterer Studieneinheiten wie Daten-Monitoring und biometrisches Zentrum. Die Aufgaben der Studienleitung umfassen insbesondere die Protokollerstellung, die Qualitätskontrolle der Durchführung, die Koordinierung der Beurteilung der klinischen Endpunkte, die Erstellung von Berichten und Publikationen, die Organisation von regelmäßigen Treffen der Studiengruppe und die Finanzierungsplanung. Dessen ungeachtet behält der klinische Prüfer eines Zentrums seine hohe Verantwortung für die ordnungsgemäße Durchführung der Studie (Korrektheit der Daten, Patientenaufklärung und -einwilligung).

\section{Anforderungen an die Biometrie und das Monitoring}

Im biometrischen Zentrum einer Studie treffen letztlich alle Studiendaten ein und werden dort zusammengeführt. Als geplantes Experiment bedarf die klinische Studie einer Fragestellung und einer entsprechenden medizinischen Hypothesenbildung, welche in eine statistische Hypothesenbildung übersetzt wird. Darin liegt eine besondere Verantwortung des biometrischen Zentrums. Zu seinen Aufgaben gehören außerdem die statistische Planung, das Datenmanagement und die Kontrolle der Datenqualität und Datenanalyse sowie eine biometrische Kontrolle von Datenfluss und Datenqualität. Die Überwachung der Rekrutierungsrate und das zeitliche Einhalten des Behandlungs-, Untersuchungs- und Beobachtungsplans kann der Monitor der Studie übernehmen. Die Komplexität einer multizentrischen Studie erfordert die Erstellung von Standardarbeitsanweisungen (SOPs), welche alle anfallenden Arbeiten regeln und Funktionen und Verantwortlichkeiten der beteiligten Institutionen transparent und verbindlich festlegen [vergleiche 4].

Die Erstellung eines statistischen Auswerteplans ist Teil der Planung einer klinischen Studie; sie ist zweckmäßigerweise 
Tab. 1. Checkliste der Studienplanung für die onkologische Therapieforschung

\begin{tabular}{|c|c|c|c|c|}
\hline Grundelemente & Spezifizierung & Phase I & Phase II & Phase III \\
\hline \multirow[t]{3}{*}{ Ziele } & Primärziel & $\begin{array}{l}\text { maximal tolerierte Dosis } \\
\text { (MTD) } \\
\text { dosislimitierende Toxizität }\end{array}$ & $\begin{array}{l}\text { Wirkung am Tumor } \\
\text { Response }\end{array}$ & $\begin{array}{l}\text { Wirksamkeitsnachweis } \\
\text { im Krankheitsverlauf } \\
\text { Überleben }\end{array}$ \\
\hline & Sekundärziel & $\begin{array}{l}\text { Gesamttoxizität } \\
\text { Pharmakokinetik } \\
\text { Wirkung am Tumor }\end{array}$ & $\begin{array}{l}\text { Toxizität } \\
\text { Überleben }\end{array}$ & $\begin{array}{l}\text { Toxizität } \\
\text { progressionsfreie Zeit } \\
\text { Lebensqualität }\end{array}$ \\
\hline & Design & $\begin{array}{l}\text { Dosiseskalationsschema } \\
\text { Bayes-Design (CRM) }\end{array}$ & 2(-3)-Stufen-Design & $\begin{array}{l}\text { Parallel-Gruppenver- } \\
\text { gleich auf Unterschied } \\
\text { oder Äquivalenz } \\
\text { gruppensequentiell }\end{array}$ \\
\hline Patientenauswahl & $\begin{array}{l}\text { Patientenpopulation } \\
\text { Vorbehandlung }\end{array}$ & $\begin{array}{l}\text { austherapiert } \\
\text { dokumentieren }\end{array}$ & $\begin{array}{l}\text { strenger Ein-/Ausschluss } \\
\text { oft «second und third line» }\end{array}$ & $\begin{array}{l}\text { aus der klinischen Praxis } \\
-\end{array}$ \\
\hline Behandlung & $\begin{array}{l}\text { Behandlungsweg } \\
\text { Behandlungsdauer und Zyklen } \\
\text { Behandlungsmodifikation(en) } \\
\text { Compliance } \\
\text { Begleitbehandlung } \\
\text { Folgebehandlung }\end{array}$ & $\begin{array}{l}\text { i.v. oder oral } \\
\text { kurz, oft nur } 2 \text { Zyklen } \\
\text { - } \\
\text { stationäre Überwachung } \\
\text { dokumentieren } \\
\text { nicht von Interesse }\end{array}$ & $\begin{array}{l}\text { unter Umständen komplex } \\
\text { bis Responsebeurteilung } \\
\text { Dosismodifikation abhängig } \\
\quad \text { von Toxizität } \\
\text { engmaschige Kontrolle } \\
\text { dokumentieren } \\
\text { nicht von Interesse }\end{array}$ & $\begin{array}{l}\text { praxisnah und durchführbar } \\
\text { oft } 1 / 2 \text { Jahr und länger } \\
\text { Dosismodifikation Abbruch } \\
\text { bei Progression } \\
\text { Maßnahmen zur Vermeidung } \\
\text { auswerten } \\
\text { auswerten }\end{array}$ \\
\hline Wirksamkeit & $\begin{array}{l}\text { Heilung bzw. Überlebenszeit } \\
\text { Symptom/Tumorfreiheit } \\
\text { Lebensqualität } \\
\text { Surrogatvariable }\end{array}$ & $\begin{array}{l}\text { nicht von Interesse } \\
\text { nicht von Interesse } \\
\text { nicht von Interesse } \\
\text { _ }\end{array}$ & $\begin{array}{l}\text { deskriptive Statistik } \\
\text { konfirmative Statistik } \\
\text { unter Umständen } \\
\text { _ dokumentieren }\end{array}$ & $\begin{array}{l}\text { konfirmative und } \\
\text { multivariate Statistik } \\
\text { deskriptive Statistik } \\
\text { konfirmative Statistik, } \\
\text { wenn doppelblind } \\
\text { Response, Biomarker }\end{array}$ \\
\hline Toxizität & $\begin{array}{l}\text { Kriterienkatalog } \\
\text { Kausalität } \\
\text { Verlaufsbeurteilung }\end{array}$ & $\begin{array}{l}\text { Auswahl aus CTC } \\
- \\
\text { kumulative Toxizität }\end{array}$ & $\begin{array}{l}\text { alle CTC Symptome beurteilen } \\
- \\
\text { deskriptive Auswertung }\end{array}$ & $\begin{array}{l}\text { komplette Sicherheitsanalyse } \\
\text { durch Randomisation } \\
\text { Auswertung in statistischen } \\
\text { Modellen }\end{array}$ \\
\hline Studienabschluss & $\begin{array}{l}\text { Abschluss der Behandlung } \\
\text { Abschluss der Studie } \\
\text { Nachsorgeplan }\end{array}$ & $\begin{array}{l}\text { im Allgemeinen nach } 2 \\
\text { Zyklen und bei Progress } \\
\text { nach Erreichen der MTD } \\
\text { - }\end{array}$ & $\begin{array}{l}\text { im Allgemeinen nach } 6 \text { Zyklen } \\
\text { und bei Progress } \\
\text { nach Stufenplan } \\
\text { - }\end{array}$ & $\begin{array}{l}\text { nach Studienplan und bei } \\
\text { Progress } \\
\text { nach statistischem Design } \\
\text { Teil des Designs }\end{array}$ \\
\hline Schlußfolgerung & $\begin{array}{l}\text { Aussage } \\
\text { Explorative Ergebnisse } \\
\text { Risiko-Nutzen Abschätzung } \\
\text { Erkenntnisgewinn }\end{array}$ & $\begin{array}{l}\text { Dosis für Phase II } \\
\text { Toxizität, Pharmakokinetik } \\
- \\
\text { Verträglichkeit, } \\
\text { Durchführbarkeit }\end{array}$ & $\begin{array}{l}\text { «proof of principle» } \\
\text { Überlebenszeit } \\
\text { informell } \\
\text { Anwendbarkeit für Phase III } \\
\text { mögliche Kombinationstherapie }\end{array}$ & $\begin{array}{l}\text { Wirksamkeit und Sicherheit } \\
\text { Praktikabilität } \\
\text { formale Auswertung } \\
\text { Zulassung } \\
\text { neuer Therapiestandard }\end{array}$ \\
\hline
\end{tabular}

unterteilt in beschreibende statistische Auswertung (Erstellung von Tabellen und Grafiken), konfirmatorische statistische Auswertung (Signifikanztests, eventuell ergänzt um die Schätzung der Größe der Wirkung einschließlich Konfidenzintervall) und explorative statistische Auswertungen (Untergruppenauswertungen, multivariate Analysen, Regressionsauswertungen).

\section{Phase I, II und III}

In der Onkologie hat sich ein Schema der klinischen Forschung und Prüfung von Medikamenten durchgesetzt, das in vier aufeinanderfolgenden Phasen erfolgt, wobei auf die Phase IV (Studie nach Zulassung) im Folgenden nicht eingegangen werden soll. Tabelle 1 zeigt zusammenfassend die we- 
sentlichen Elemente der Phase-I-, Phase-II- und Phase-IIIStudien, an denen sich Anforderungen und Qualität dieser Schritte der Therapieforschung festmachen lassen.

Das dreiphasige Konzept klinischer Erkenntnisgewinnung bildet auch die Grundlage für jede Prüfung neuer Substanzen und neuer Wirkstoffe zum Zweck ihrer Zulassung. Zulassung kann nur erteilt werden, wenn Wirksamkeit und Sicherheit einer neuen Substanz oder einer neuen Behandlungsform gezeigt und belegt werden kann. Dazu gehört in der Onkologie auch die neuartige Kombination von Substanzen, die ihre Wirksamkeit und Sicherheit einzeln schon bewiesen haben sowie die Erweiterung der Indikation von zugelassenen Medikamenten auf andere Tumoren. Ausschlaggebend für eine $\mathrm{Zu}-$ lassung sind eindeutige, nachvollziehbare und gültige Ergebnisse aus den klinischen Phasen I-III, wobei der Wirksamkeitsprüfung in der großen, randomisierten, kontrollierten Phase-III-Studie immer noch die entscheidende Funktion zukommt. Die Forderung nach der Vorlage von mindestens zwei unabhängigen positiven Phase-III-Studien für eine Zulassung ist in der Praxis der Zulassungsbehörden teilweise abgeschwächt worden. So kann eine gute Phase-III-Studie ausreichen, wenn anderweitige klinische Evidenz, z.B. durch gute überzeugende Phase-II-Studien, für die Wirksamkeit gezeigt werden kann und auch die präklinischen Ergebnisse überzeugen. Wichtig ist jedoch der Aspekt der Wiederholbarkeit unter unabhängigen Gegebenheiten, welcher bei der Planung der Substanzentwicklung und ihrer klinischen Prüfung mit dem Ziel der Zulassung sorgfältig einzuplanen ist.

\section{Phase I}

Ziel der Phase-I-Studie ist die Dosisfindung für eine neue Substanz. Diese erfolgt als Bestimmung einer maximal tolerierten Dosis (MTD) verbunden mit einer vollständigen Dokumentation aller aufgetretenen unerwünschten Ereignisse und ergänzt durch pharmakokinetische Untersuchungen. Das praktische Ziel ist der Vorschlag einer Dosis für die weitere klinische Prüfung. Erste Wirkungen werden dokumentiert. Die Phase-I-Untersuchung erfolgt nach einem prospektiven Studienprotokoll an austherapierten Patienten mit fortgeschrittener Krebserkrankung, wobei oft eine breite Klasse von Tumoren zugelassen wird. Die Behandlung erfolgt nach einem statistisch abgesicherten Stufenplan [vergleiche 5, 6] und erfordert ein hohes Maß an Spezialisierung und Koordination. Die Phase-I-Studie kann deswegen nur an ausgewählten Studienzentren durchgeführt werden. Ihre Fallzahl bewegt sich meist im Bereich von 20-60 Patienten. Neuere Phase-I-Designs benutzen die statistische Methodik eines fortlaufenden Beurteilens der MTD («continual reassessment method»; CRM) und versprechen eine wesentliche Reduktion der Fallzahl bei gleichzeitig optimaler Behandlung in der Nähe einer möglicherweise wirksamen Dosis. Die Behandlung eines Patienten sollte über mindestens zwei Zyklen möglich sein.
Dosissteigerungen innerhalb der Behandlung eines Patienten bedürfen eines speziellen Studienplans.

\section{Phase II}

Die Phase-II-Studie wendet prospektiv ein bestimmtes Dosisschema mit strengen Ein/-Ausschlusskriterien an. Sie hat das Ziel, die therapeutische Aktivität einer Behandlung grundsätzlich nachzuweisen. Liegen verschiedene Behandlungen zur Prüfung vor, sollten diese randomisiert in parallel durchgeführten Phase-II-Studien geprüft werden, in denen aber wegen der begrenzten Fallzahl der Parallelgruppenvergleich nicht das Hauptziel sein kann, sondern dem Wirksamkeitsvergleich der Phase III vorbehalten ist. Entsprechend kann bei Prüfung einer Substanz eine parallele Kontrollgruppe mit Standardbehandlung mitgeführt werden. Unter verschiedenen statischen Designs haben sich die Mehrstufenpläne und unter diesen der optimale Zweistufenplan von Simon in der Praxis bewährt [7]. Nicht vorbehandelte Patienten können nur nach sorgfältiger Prüfung und Begründung in eine Phase-II-Studie aufgenommen werden. Erforderlich ist dann die Erwartung eines therapeutischen Nutzens der neuen Substanz, welche die Vorenthaltung einer Standardbehandlung rechtfertigt. Da es sich um eine grundsätzliche Prüfung auf Wirksamkeit handelt, sollte die Patientengruppe möglichst homogen sein, um die interindividuelle Variabilität gering zu halten. Patienten sollten auf jeden Fall messbare Tumorläsionen aufweisen, und die Methoden ihrer Messung müssen im Studienprotokoll genauestens festgelegt sein. Durch die Einführung der RECIST-Kriterien [8] wurde eine Revision der WHO-Kriterien [9] eingeleitet, welche der Kontrolle der Progressionsrate mehr Gewicht verleiht, um Medikamenten Rechnung zu tragen, die das Fortschreiten der Erkrankung wesentlich verlangsamen und so die Überlebensrate verlängern können. Neben dem Nachweis der Aktivität einer Behandlung kommt in einer Phase-II-Studie der Beurteilung der Toxizität eine ganz besondere Bedeutung zu. In ihrem Ergebnis muss die Phase-IIStudie therapeutischen Nutzen und Toxizitätsrisiko abwägen.

\section{Phase III}

Im Rahmen eines klinischen Forschungsvorhabens hat die randomisierte Phase-III-Studie die Aufgabe, die in einer Phase-II-Studie gefundene Wirksamkeit zu bestätigen und die relative Wirksamkeit im Vergleich zu Therapien mit nachgewiesener Wirksamkeit zu bestimmen (Überlegenheit) oder eine Äquivalenz zu etablierten Therapien zu zeigen. Eine Placebokontrolle ist in der Onkologie eher selten und muss im Einzelfall gut begründet und ethisch sorgfältig geprüft werden. Die typische Zielgröße der Wirksamkeit einer onkologischen Phase-III-Studie ist die Überlebenszeit. Sie kann parametrisiert beurteilt werden mittels der medianen Überlebens- 
zeit oder, mittels Überlebensraten nach einigen Jahren (1-Jahresrate, 5-Jahresrate usw.), wird aber statistisch stets in ihrem gesamten Verlauf mittels Überlebenskurven (Kaplan-MeierKurven, Log-rank-Test) beurteilt. Plant man Überleben, Progression und Lebensqualität als primäre Zielgrößen, so stellen diese drei multiple Endpunkte dar, was bei der Planung zu berücksichtigen ist. Die Fallzahlen einer randomisierten klinischen Studie bewegen sich in einem Bereich von mehreren hundert Patienten pro Therapiearm. Die Phase III prüft insbesondere neue Kombinationen von Behandlungen/Substanzen. Dies erfordert große Studien, da oftmals die Komponenten der Kombination als Parallelgruppen randomisiert mitgeführt werden müssen.

\section{Anforderung an die Phase-III-Studie: Randomisation und Stratifikation}

In der randomisierten klinischen Studie werden Patienten nach einem Zufallsmechanismus den Behandlungsarmen so zugeteilt werden, dass jeder Patient die gleiche Chance besitzt, eine der zur Auswahl stehenden Behandlungen zu bekommen. Die Randomisation verhindert mittels so erzwungener Gleichheit in Struktur, Beobachtung und Behandlung (außer der Prüfbehandlung) eine Selektionsverzerrung des Therapievergleichs, die z.B. dadurch zustande kommen kann, dass einer Behandlungsgruppe prognostisch günstigere $\mathrm{Pa}$ tienten zugeteilt werden. Der beobachtete Unterschied kann somit als kausaler Behandlungsunterschied interpretiert werden. Die Randomisation sollte als Telefon- oder Fax-Randomisation in einem unabhängigen Zentrum, in der Regel dem biometrischen Zentrum der Studie, durchgeführt werden. Ethische Voraussetzung ist eine Vergleichbarkeit und eine Gleichwertigkeit der zu prüfenden Therapie («equipose») mit der Kontrollbehandlung.

Mit einer Stratifikation, auch Blockbildung oder Schichtung genannt, wird die Wirkung der Prüftherapie von derjenigen bekannter Störgrößen getrennt beurteilbar, insbesondere wird aber die Wirkung der Therapie und ihre Wechselwirkung mit möglichen bekannten Störgrößen auswertbar und interpretierbar. Die Randomisation erfolgt dann in jedem Stratum getrennt. Grundsätzlich sollte jede Klinik ein Stratum bilden.

\section{Anforderung an die Phase-III-Studie: Sequentieller Auswerteplan}

Das Grunddilemma aller Datenauswertungen vor der Endauswertung (Zwischenauswertungen) liegt darin, dass die Wahrscheinlichkeit in einer von mehreren Zwischenauswertungen unter der Nullhypothese ein signifikantes Ergebnis zu finden desto größer wird, je mehr Zwischenauswertungen durchführt werden. Mit einer Adjustierung des Signifikanzniveaus kann dies behoben werden. Auf der anderen Seite gibt es ethische Gründe, eine Studie dann abzubrechen, wenn ein
Therapiearm dem anderen unterlegen ist, was nur in einer Zwischenauswertung nachgewiesen werden kann. Dazu wurden verschiedene statistische Verfahren konzipiert. Gruppensequentielle Pläne und Pläne mit wiederholtem Signifikanztest erlauben statt nur einem Test (nach Abschluss und Erreichen der geplanten Gesamtzahl) wiederholte Signifikanztests im Laufe der Studie. In onkologischen Studien mit der Überlebenszeit als Endpunkt sind Zwischenauswertungen nur bedingt einsatzfähig wegen der langen Zeit bis zur Evaluation des Endpunktes «Tod». Neuere Verfahren versuchen, ein sequentielles Auswertekonzept von Beginn an zu etablieren und halten die Fallzahl der Studie offen [10, 11]. Die Ergebnisse von Zwischenauswertungen sind vom methodischen Standpunkt aus stets kritisch zu betrachten, da ein Ergebnis einer Zwischenauswertung grundsätzlich nicht das protokollgemäße Studienergebnis ersetzen kann, für das die Studie geplant und das zwischen Studienleitung, Prüfzentren und Patienten vereinbart war.

\section{Diskussion}

Mit den Regeln der Good Clinical Practice (GCP) ist ein Regelwerk für die Durchführung klinischer Studien entstanden, das einen hohen Qualitätsstandard der klinischen Forschung garantieren soll. Vorrangig gewidmet dem Schutz und den Persönlichkeitsrechten des Patienten und der Qualität der Daten des Wirksamkeitsnachweises, hat sich GCP zu einer umfassenden Richtlinie entwickelt. GCP-Regeln für Ausschluss von Patienten aus der Auswertung fordern ein prospektives Vorgehen. Über einen Ausschluss soll blind entschieden werden. In jedem Fall ist die ausgeschlossene Population separat zu dokumentieren und in der Publikation zu berücksichtigen. Deswegen sollte die Prüfung der Ein-/ Ausschlusskriterien bei der Patientenaufnahme sehr ernst genommen werden. Patienten, die nicht nach Plan behandelt wurden, sind ebenfalls zu beschreiben. GCP verlangt eine Festlegung der schlussendlichen statistischen Auswertung im statistischen Teil des Studienprotokolls, und es wird auf einen $\mathrm{zu}$ erstellenden statistischen Auswerteplan abgehoben, der nur solange ergänzt werden sollte, wie die klinischen Prüfer und Auswerter noch blind gegenüber der Therapiezuweisung sind.

Die «Gesetze» der GCP bilden heute die Grundlage für die klinische Zulassung und werden zu diesem Zweck kontinuierlich vervollständigt und weiter entwickelt.

Neue Anforderungen sind für solche Behandlungen zu entwickeln, die nicht mehr auf breiter Basis zytostatisch wirken, sondern vielmehr bestimmte Phasen des Tumorwachstums hemmen, das Immunsystem verändern oder z.B. die Tumorangiogenese hemmen. Solche zielgerichteten Medikamente zei- 
gen oftmals nur geringe Toxizitäten und stellen somit bereits die Rationale des Phase-I-Studienansatzes in Frage. Nicht mehr die MTD ist dann die primäre Zielgröße, sondern diejenige Dosis, welche einen maximalen molekularen Effekt hervorruft. Derartige Dosen können weit unter einer klinisch beobachtbaren MTD liegen.

Die behandlungsinduzierte Modulation der Genexpression in Tumorzellen stellt eine neue Richtung der Therapieforschung dar, für die noch nicht geklärt scheint, ob sie direkt zur Verbesserung von Therapieergebnissen führt oder ob sie zunächst lediglich eine weitere und genauere Charakterisierung des Verlaufs der Tumorerkrankung liefern wird. Diese neue und in ihrem Umfang überwältigende genomische Information muss also noch zeigen, ob sie eine für die Krebserkrankung eines Patienten prognostische oder prädiktive Funktion hat.
In jedem Fall sind derartige «Begleituntersuchungen» prospektiv zu planen, und Qualitätssicherungsmaßnahmen sind zu implementieren. Bei Verwendung von Genexpressionsdaten ergibt sich eine Kaskade von Fehlerquellen, die durch ein experimentelles Design kontrolliert werden müssen. Eine wichtige Anforderung ist deswegen, dass die unterschiedlichen klinischen und experimentellen Teams optimal nach besonderen SOPs zusammenarbeiten.

\section{Dank}

Als Biometriker möchte ich bei dieser Gelegenheit Wolfgang Queißer danken für eine zwanzigjährige enge und gute interdisziplinäre $\mathrm{Zu}$ sammenarbeit und die Möglichkeit der Mitarbeit in vielen seiner klinischen Studien.

\section{Literatur}

1 Chow S-C, Liu J-P: Design and Analysis of Clinical Trials. New York, Wiley, 1998.

2 Pocock S: Clinical Trials. Chichester, Wiley, 1983.

3 Senn S: Statistical Issues in Drug Development. Chichester, Wiley, 1997.

4 Kreuser ED, Fiebig HH, Scheulen ME, Hanauske A, Keppler BK, Mross K, Schalhorn A, Eisenbrand G, Edler L, Höffken K, Berdel WE: Standard Operating Procedures and Organization of German Phase I, II and III Study Groups, New Drug Development Group (AWO) and Study Group of Pharmacology in Oncology and Hematology (APOH) of the Association for Medical Oncology (AIO) of the German Cancer Society. Onkologie 1998;21 (suppl 3):1-22.
5 Hanauske AR, Edler L: New clinical trial designs for phase I studies in hematology and oncology: Principles and practice of the continual reassessment model. Onkologie 1996;19:404-406.

6 Edler L: Overview of phase I trials; in Crowley J (ed): Handbook of Statistics in Clinical Oncology. New York, Marcel Dekker, 2001, pp 1-34.

7 Edler L: Phase II Studien in der Onkologie: Wie viele Patienten sind erforderlich? Tumordiagn Ther 1998;14:1-9.

8 Therasse P, Arbuck SG, Eisenhauer EA, Wanders J, Kaplan RS, Rubinstein L, Verweij J, Van Glabbeke M, van Oosterom AT, Christian MC, Gwyther SG: New guidelines to evaluate the response to treatment in solid tumors. J Natl Cancer Inst 2000;92: 205-216.
9 WHO: WHO Handbook for Reporting Results of Cancer Treatment. Geneva, World Health Organization, 1979.

10 Bauer P, Köhne K: Evaluation of experiments with adaptive interim analyses. Biometrics 1994;50: 1029-1041.

11 Hartung I: A self-designing rule for clinical trials with arbitrary response variables. Control Clin Trials 2001;22:111-116. 